Article

\title{
A Tidal Flat Wetlands Delineation and Classification Method for High-Resolution Imagery
}

\author{
Hong Pan $\left.{ }^{+} \mathbb{(}\right)$, Yonghong Jia ${ }^{*}{ }^{\dagger}$, Dawei Zhao, Tianyu Xiu and Fuzhi Duan \\ School of Remote Sensing and Information Engineering, Wuhan University, Wuhan 430079, China; \\ panhong@whu.edu.cn (H.P.); zhaodawei@whu.edu.cn (D.Z.); xiutianyu@whu.edu.cn (T.X.); \\ duanfz@whu.edu.cn (F.D.) \\ * Correspondence: 00200242@whu.edu.cn \\ † These authors contributed equally to this work.
}

Citation: Pan, H.; Jia, Y.; Zhao, D.; Xiu, T.; Duan, F. A Tidal Flat Wetlands Delineation and Classification Method for High-Resolution Imagery. ISPRS Int. J. Geo-Inf. 2021, 10, 451. https://doi.org/10.3390/ijgi10070451

Academic Editors: Jamal Jokar Arsanjani and Wolfgang Kainz

Received: 9 May 2021

Accepted: 26 June 2021

Published: 1 July 2021

Publisher's Note: MDPI stays neutral with regard to jurisdictional claims in published maps and institutional affiliations.

Copyright: (c) 2021 by the authors. Licensee MDPI, Basel, Switzerland. This article is an open access article distributed under the terms and conditions of the Creative Commons Attribution (CC BY) license (https:// creativecommons.org/licenses/by/ $4.0 /)$

\begin{abstract}
As an important part of coastal wetlands, tidal flat wetlands provide various significant ecological functions. Due to offshore pollution and unreasonable utilization, tidal flats have been increasingly threatened and degraded. Therefore, it is necessary to protect and restore this important wetland by monitoring its distribution. Considering the multiple sizes of research objects, remote sensing images with high resolutions have unique resolution advantages to support the extraction of tidal flat wetlands for subsequent monitoring. The purpose of this study is to propose and evaluate a tidal flat wetland delineation and classification method from high-resolution images. First, remote sensing features and geographical buffers are used to establish a decision tree for initial classification. Next, a natural shoreline prediction algorithm is designed to refine the range of the tidal flat wetland. Then, a range and standard deviation descriptor is constructed to extract the rock marine shore, a category of tidal flat wetlands. A geographical analysis method is considered to distinguish the other two categories of tidal flat wetlands. Finally, a tidal correction strategy is introduced to regulate the borderline of tidal flat wetlands to conform to the actual situation. The performance of each step was evaluated, and the results of the proposed method were compared with existing available methods. The results show that the overall accuracy of the proposed method mostly exceeded $92 \%$ (all higher than $88 \%$ ). Due to the integration and the performance superiority compared to existing available methods, the proposed method is applicable in practice and has already been applied during the construction project of Hengqin Island in China.
\end{abstract}

Keywords: tidal flat wetlands; high-resolution images; shoreline prediction; feature descriptor; tidal correction

\section{Introduction}

As a transition zone between terrestrial and marine ecosystems, coastal wetlands play an important role in resisting coastal erosion, avoiding sea level rise, preserving shorelines, and so on [1-3]. As a significant component of coastal wetlands, tidal flat wetlands contribute a lot to their ecological functions. Due to the acceleration of urbanization and the intensification of human activities, however, some unreasonable coastal utilization and offshore pollution have emerged, which have accelerated the erosion and deterioration of tidal flat wetlands and seriously threatened the coastal ecosystem [4]. Therefore, the monitoring and delineation of tidal flat wetland degradation is an urgent task, an important part of which comprises extracting tidal flat wetlands [5]. Tidal flat wetlands are distributed in intertidal zones [6], with three main categories: rocky marine shore (RMS), sand marine shore (SMS), and mud marine shore (MMS).

Areas of tidal flats vary greatly between different regions, resulting in inconsistent extraction target sizes, while the research targets are all essential no matter their size. Therefore, the resolution of remote sensing images should be adequately high to satisfy 
the extraction demands. On the other hand, it is no longer a problem to obtain highresolution images such as WorldView-2 (WV-2) with the development of aerospace remote sensing technology $[7,8]$, which guarantees the effective resolution of the data source. After comprehensive consideration, it is significant to propose a method for tidal flat wetland extraction from high-resolution images [9].

Although there are no specific remote sensing methodologies for tidal flat wetland extraction, numerous extraction methods have been applied to convert remote sensing data into other land cover maps. A general method comprises combining mathematical statistics with manual interpretation. However, it is inefficient, with low accuracy, intensive labor, and dependence on human analysis. Multiple intelligent methods have been recommended in image extraction, such as multisource information fusion technology used in remote sensing mapping. Synthesis of spectral features $[10,11]$ can distinguish targets by different brightness levels between image bands, classification by the fusion of different texture information [12] can take both the macroscopic nature and the detailed structure of targets into account, normalized difference index information $[13,14]$ contributes to eliminating the influence of the difference in topography, and the combination of expert knowledge [15] is conducive to comprehensive interpretation and decision analysis of remote sensing images. The composition technology of multisource information has achieved specific results; however, depending on multisource information exclusively is not enough to extract the research targets accurately. Therefore, the proposed method is a combination of multisource information composition and multiclassification technologies.

Traditional remote sensing image classification technology has been divided into unsupervised classification and supervised classification [16]. In unsupervised classification, statistical distance is typically used to measure the similarity, and the category intervals are maximized by distinguishing targets based on a specific algorithm $[17,18]$. When a specific set of targets is desired, supervised classification is more appropriate. The sample set of each category is selected first, a classification algorithm can be trained using the samples, and, finally, the remaining pixels are classified. The maximum likelihood classification (MLC) $[19,20]$ method is a generally used supervised classification algorithm that establishes a nonlinear discriminant function set according to the Bayesian information criterion (BIC) [21]. The support vector machine (SVM) [22-24] method is a widely used classification method in remote sensing images based on the structural risk minimization principle. The decision tree (DT) $[25,26]$ algorithm is also a supervised classification method based on spatial data mining and knowledge discovery. Prior knowledge can be used in supervised classification to select training samples, and the classification accuracies can be improved by repeatedly testing training samples. However, supervised classification methods depend on the quality of training samples excessively, which makes it inefficient to select samples with more human capital and time. Moreover, supervised classification can only identify specific categories, which is not conducive to the classification of complex targets.

In recent years, deep learning [27] methods have also been employed in the research of remote sensing information extraction. An algorithm named deep belief network (DBN) [28] has unleashed the characteristics of unsupervised learning with the identification of remote sensing features. However, the selection of network parameters requires the intervention of prior knowledge, which makes it difficult to determine the appropriate parameters. The generally used convolutional neural network (CNN) $[29,30]$ algorithm in the deep learning method has obvious advantages in processing high-dimensional images, but the scanty samples of remote sensing images have limited the generalization ability of the CNN algorithm. Another deep learning algorithm, autoencoder (AE) [31,32], still needs to be combined with other classifiers to achieve appropriate results. Compared with traditional machine learning methods, deep learning methods have stronger fitting capabilities, and they can learn beneficial features from numerous data. However, they similarly rely on training samples excessively. Researchers have to spend a lot of time and energy collecting appropriate samples. Additionally, deep learning methods require high-performance computers to achieve fast computing and have time-consuming training 
procedures, so they are not suitable for the extraction of small targets with limited samples such as shore targets. Therefore, it is necessary to design a comprehensive and complete extraction method for shores considering the inadequacy of existing methods.

The aim of this study is to propose a method specifically for tidal flat wetland delineation and classification from high-resolution images and evaluate the method. First, remote sensing features and geographical buffers are used to establish a decision tree for initial classification. Next, a natural shoreline prediction algorithm is designed to refine the range of the tidal flat wetland. Then, a range and standard deviation descriptor is constructed to extract the rock marine shore, a category of tidal flat wetlands. A geographical analysis method is considered to distinguish the other two categories of tidal flat wetlands. Finally, a tidal correction strategy is used to regulate the borderline of tidal flat wetlands to conform to the actual situation. The performance of each step is evaluated, and the results of this method were compared with existing available methods to illustrate the effectiveness and practicability of the proposed method. As a first application, this method is demonstrated in Hengqin Island (an island in China facing directly to the South China Sea). A better understanding of the distribution of shores in Hengqin Island is critical to wetland protection and construction planning.

\section{Methods}

\subsection{Overall Processes}

The proposed method originates from the perspective of logical layering. According to the definition of tidal flat wetlands, it belongs to natural wetlands, and there are three categories, including rocky marine shore (RMS), mud marine shore (MMS), and sand marine shore (SMS). On the first logical level, the approximate range of the tidal flat wetland was extracted, which is regarded as the initial classification. Then, the range was refined on the second logical level by predicting the natural shore edges. Finally, the target objects were reclassified on the third logical level to obtain the three wetland categories. The classification system and the logical structure are shown in Figure 1. The overall flowchart is shown in Figure 2, and the procedures are summarized as follows.

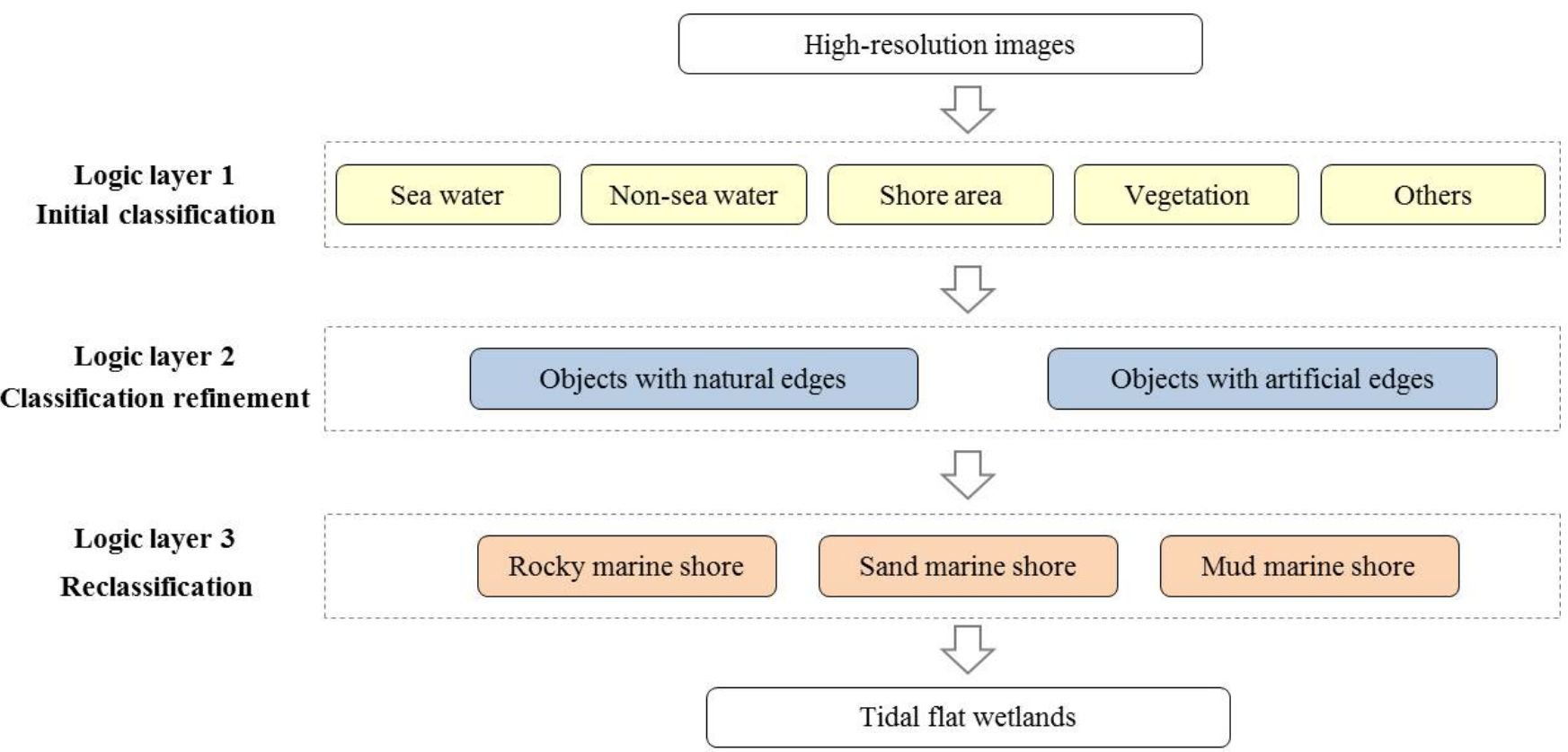

Figure 1. Logical structure of this study. 


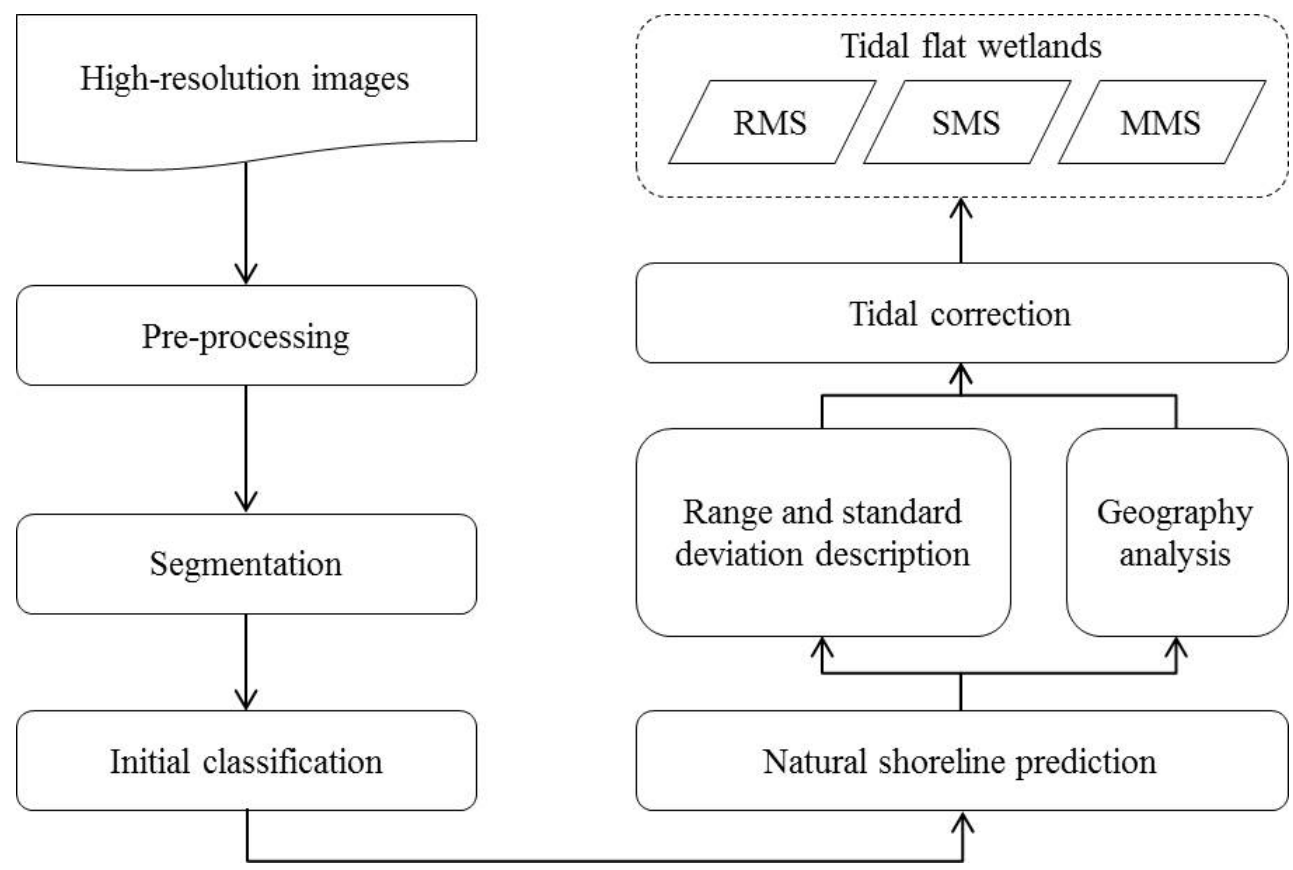

Figure 2. Overall flowchart of the proposed method.

\subsubsection{Preprocessing}

Radiation calibration, geometric correction, data fusion, and segmentation are included in this step. In order to minimize the local differences of image objects and make the remote sensing features of each category prominent, multiresolution segmentation achieved by the fractal net evolution approach (FNEA) was conducted on the research images before classification via the software eCognition Developer 9.0. After repeated experiments, three segmentation parameters of FNEA were arranged: the scale parameter was 200 , the shape index was 0.1 , and the compactness was 0.5 . The categories involved in this study could be distinguished optimally with the parameters above.

\subsubsection{Initial Classification}

The purpose of this step is to perform initial classification on the high-resolution images to extract the shore area roughly, i.e., accomplish Logic Layer 1 in Figure 1. There is both water and vegetation in the targets to be classified; therefore, it is important for the remote sensing features to be sensitive to water and vegetation. Normalized difference indexes are often used in the classification of remote sensing images because they are easy to compare, for which reason we chose the commonly used NDWI and NDVI to distinguish water and vegetation. Considering the adjacent distance between shore and sea, we divided the water into seawater and non-seawater according to the acreage of water targets, and the buffer zone of seawater targets was constructed to restrict the distribution of targets. The features explored in this study and their descriptions are shown in Table 1.

Table 1. Summary of the features explored in this study and their descriptions.

\begin{tabular}{lr}
\hline Name & Description \\
\hline NDWI & (RGREEN-RNIR)/(RGREEN + RNIR) [33] \\
NDVI & (RNIR-RRED)/(RNIR + RRED) [34] \\
$\begin{array}{c}\text { Area } \\
\text { Buffer }\end{array}$ & Pixel numbers of target object multiply area of every single pixel \\
$\begin{array}{l}\text { where RGREEN, RNIR, and RRED are the reflectance values in the green (Band 2), NIR (Band 4) and red (Band 3) } \\
\text { bands, respectively. }\end{array}$
\end{tabular}

First, we sampled the categories to be classified, and then we calculated the NDWI of each sample. In this study, the sample number of each category is 200, and the NDWI 
value lines are shown in Figure 3a. $T_{W}$ represents the optimal threshold, which maximizes the distinction between water and nonwater. It can be observed that water and nonwater objects can be distinguished when $T_{W}$ ranges from 0 to 0.5 . To obtain the specific value, we calculated the classification deviations (the total deviation of the misclassified samples in the target category and the nearest nontarget category) in this range, as shown in Figure $3 \mathrm{~b}$. The minimum deviation exists when the NDWI equals 0.11. Similarly, $T_{V}$ represents the optimal threshold, which maximizes the distinction between vegetation and nonvegetation objects, and the value of $T_{V}$ can be determined as 0.65, as shown in Figure 4 . In addition, $S_{\max }$ represents the merged water object with the largest area. The decision tree was established, as shown in Figure 5, and the classification was executed based on the rules. In order to improve the initial classification results, it was necessary to merge the connected objects and remove the small patches. Then, the rough scope of shore areas could be obtained.

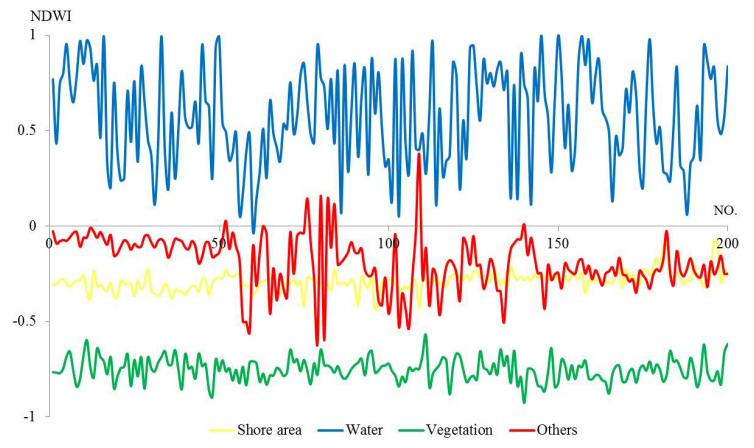

(a)

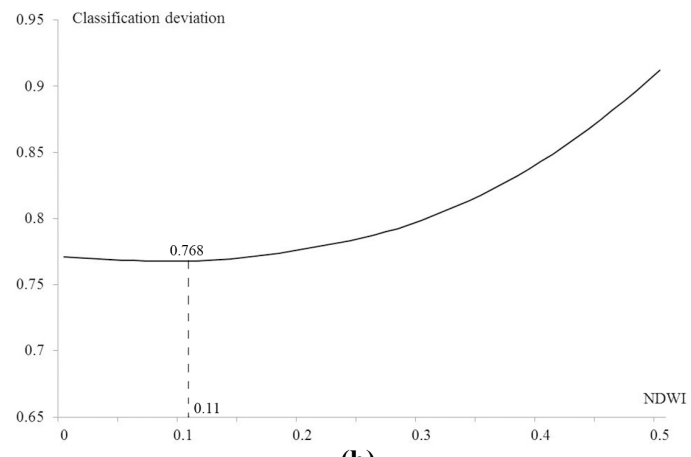

(b)

Figure 3. Determination of $T_{w}$. (a) NDWI line of each category. (b) Deviation trend with the NDWI value changing from 0 to 0.5 .

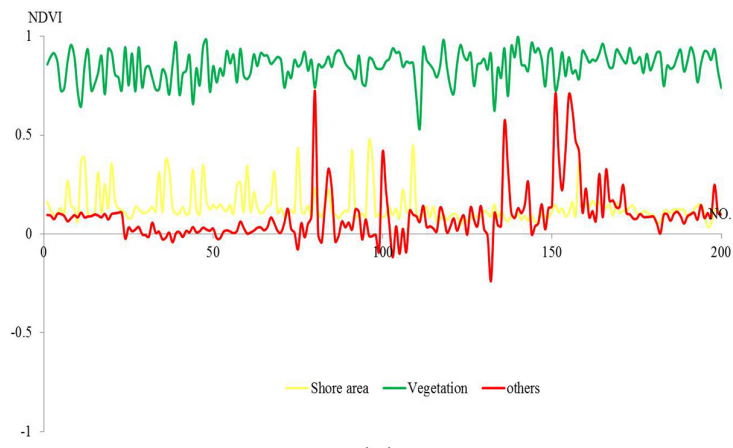

(a)

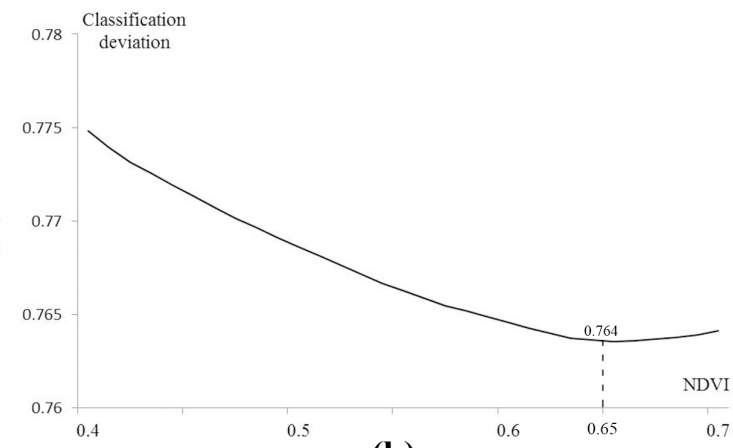

(b)

Figure 4. Determination of $T_{v}$. (a) NDVI line of each category. (b) Deviation trend with the NDVI value changing from 0.4 to 0.7 . 


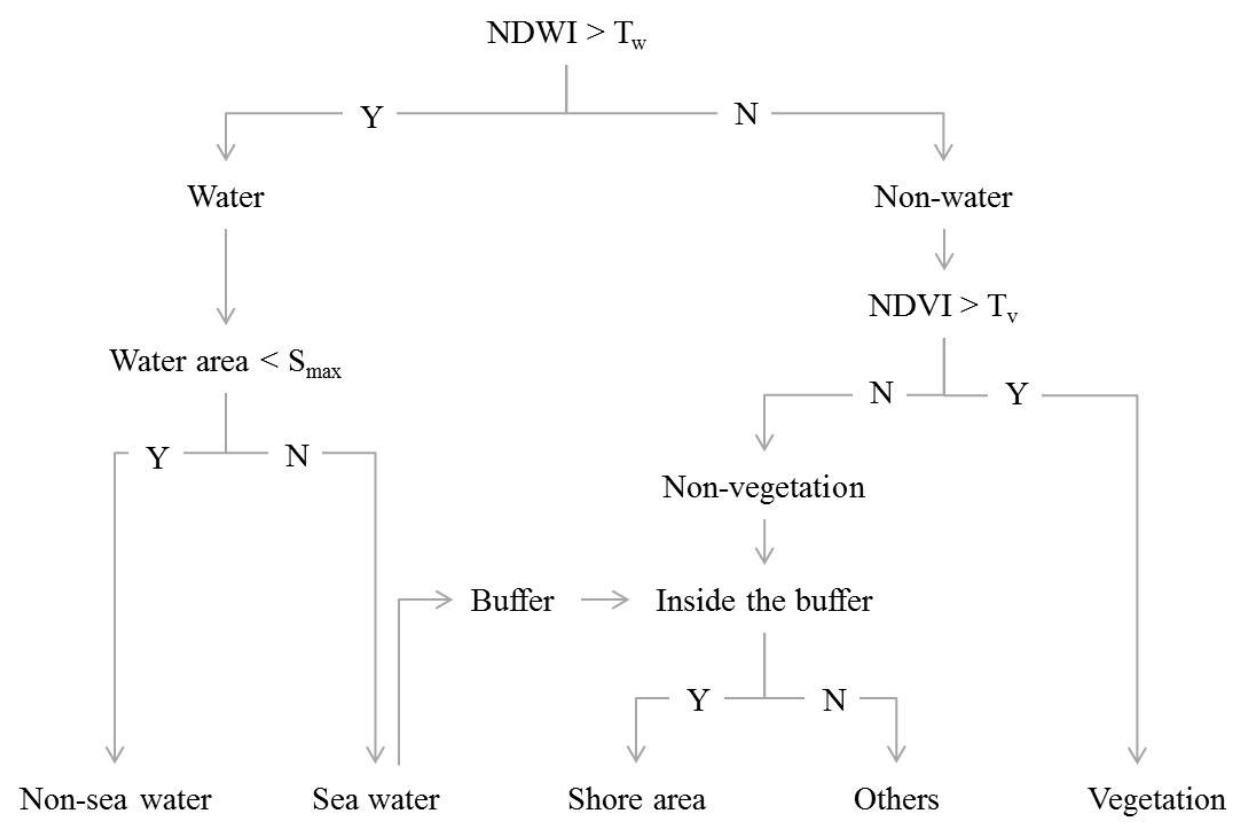

Figure 5. Decision tree for the initial classification.

\subsubsection{Classification Refinement}

The purpose of this step is to refine the range of tidal flat wetlands. The rough scopes of shore areas obtained in the initial classification contained both natural boundaries and artificial ones, while the latter did not belong to tidal flat wetlands. To predict the natural boundaries to refine the tidal flat wetlands, an algorithm based on the slope change of target edges was designed and is explained in Section 2.2 in detail.

\subsubsection{Reclassification}

In this step, reclassification was conducted to extract the RMS, MMS, and SMS. First, a new feature descriptor based on the range and standard deviation was designed to extract the RMS. Then, a geographical analysis method was utilized to classify the MMS and SMS. Finally, a tidal correction method was applied to predict the low tide beach line of tidal flat wetlands in order to obtain the complete wetland targets. The range/standard deviation description method and the tidal correction method will be described in detail from Sections 2.3 to 2.4. The geographical analysis method applied to distinguish the MMS and SMS will be explained in this section.

The composition of the SMS is similar to the MMS, and their slight differences lie in the sizes of sand or mud particles, which are usually distinguished by manual exertion. Limited by resolution, it is still a problem to distinguish them by remote sensing features, even in high-resolution images. Fortunately, the SMS and MMS have obvious discriminations according to their causes and distributions. The SMS is generally formed of stones being sharpened into small particles by waves, so they are mostly distributed in the shore areas directly towards the ocean with strong wave effects. The MMS is formed by sediments of mud. They are distributed in areas with slow currents and weak wave effects, such as lagoons and estuaries. Therefore, the geographical analysis method is proposed to distinguish the SMS and MMS.

Hengqin Island, to which the study area belongs, is an island towards lands from the north, west, and east sides, but only the south side points directly towards the South China Sea. In Image 1, the SMS and MMS are distributed around an obvious lagoon and the south shore area with strong wave effects. According to the geographical causes and definitions above, we could scope the distribution range of the two shore wetlands. The SMS was distributed around the lagoon, and the MMS was distributed in the south shore area. As a result, the shore wetlands were distinguished, as shown in Figure 6a. In Image 2, 
natural shores are all distributed in the south areas towards the ocean, which means there are no appropriate circumstances for the MMS. Therefore, the natural shores in Image 2 were all classified into SMS instead of RMS, as shown in Figure 6b.

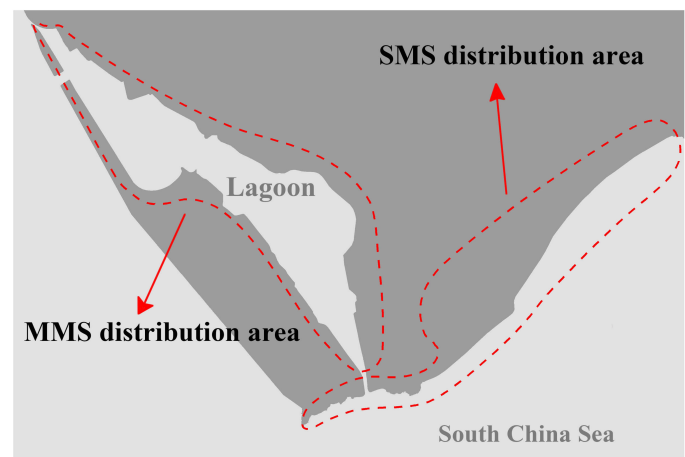

(a)

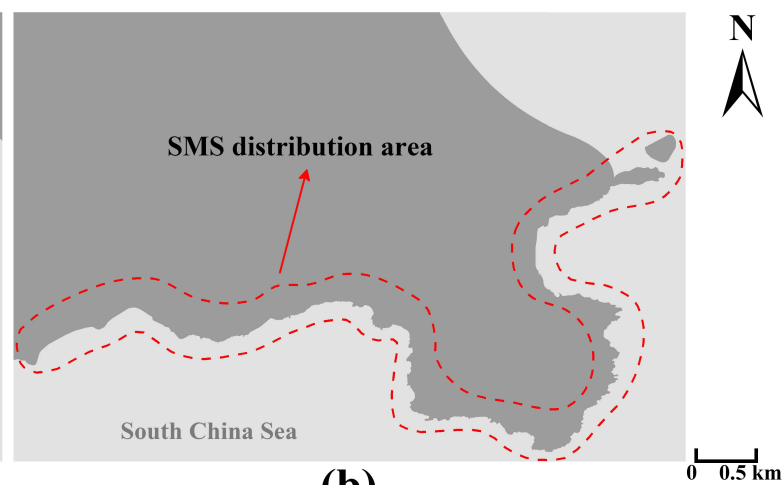

(b)

Figure 6. The geographical analysis method used to distinguish the SMS and MMS. (a) Geographical analysis in Image 1. (b) Geographical analysis in Image 2.

\subsection{Natural Shoreline Prediction}

Artificial shorelines are often difficult to distinguish from natural ones because of their similar spectral or texture features. Considering that edges of artificial shores are usually regular and the natural shores are not, a natural shoreline prediction (NSP) algorithm based on the slope change rate of edges was designed.

For the rough shore area extracted in the initial classification, the shore polygon edge adjacent to the sea water was defined as a line vector named $L$, and $L$ was equally divided into $n$ sections from $l_{1}$ to $l_{n}$. Then, the slope of each $l$ as $K_{i}$ was calculated

$$
K_{i}=\frac{y_{e}-y_{s}}{x_{e}-x_{s}}
$$

where $\left(x_{s}, y_{s}\right)$ and $\left(x_{e}, y_{e}\right)$ represent the start point and the end point of each section, respectively.

The serial number of $l$ is selected as the abscissa, and the slope of $l$ is selected as the ordinate to obtain a slope change curve $y=f(k)$. The change rate of $K_{i}$ as $K_{i}^{\prime}$ was calculated, after which the slope change rate curve could be obtained. In order to distinguish the stable section and the fluctuation section of the curve, a threshold $T$ was set $(T$, defined as onetwentieth of the difference between the max and the minimal $K_{i}^{\prime}$ ), and when $K_{i}^{\prime} \notin[-T, T]$, $l$ was predicted as a natural shoreline. The steps of the proposed NSP algorithm are summarized in Algorithm 1.

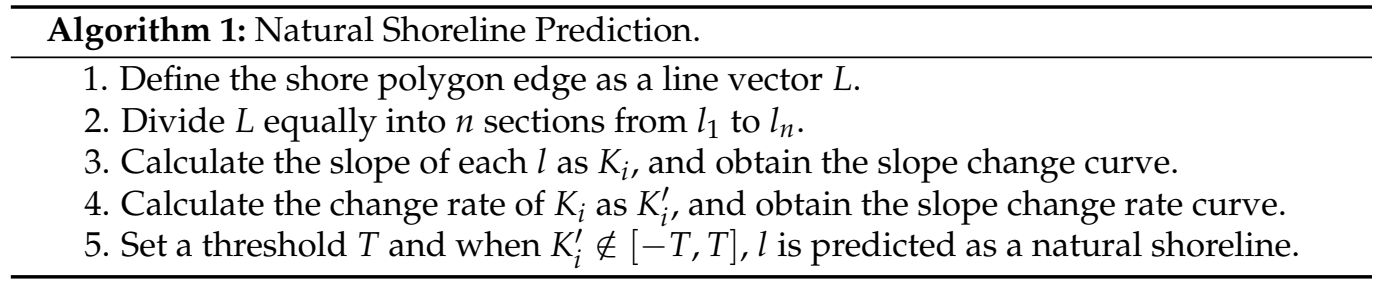

\subsection{Range and Standard Deviation Description}

In order to minimize the difference of image objects, the previous steps were based on object-oriented segmentation. However, there were two problems. On the one hand, the rock targets had different particle sizes compared to the other two targets, which means they had different textural features, and it was hard to keep the texture differences on the same scale. In this way, appropriate segmentation scales to preserve the textural 
features of both the RMS and other targets were difficult to select. On the other hand, the spectral features of the RMS, SMS, and MMS were similar, which made it troublesome to distinguish any of them, even at an object-oriented level. Considering the problems above, a pixel-based extraction method was designed and is presented in this part.

Observing the characteristics of the three shore wetlands, we found that the RMS has a more complex texture than the others, resulting in discrete gray values in a window with a certain scale. Hence, the range and standard deviation, which reflect the tightness and dispersion of the data, were selected to design a new feature description-the range and standard deviation (RSD) descriptor. Despite that high-resolution images with four bands contain plenty of information, the quantity of redundant information exists when they are employed to extract the RMS, as shown in Figure 7a. Therefore, two bands with less relevance were selected for feature description, as shown in Figure 7b. The specific steps of this method are described as follows.

(1) Clip the high-resolution images by polygons of the refined tidal flat wetlands, and reserve the blue band and the near-infrared band.

(2) Traverse each band by a window of $5 \times 5$, and calculate the standard deviation of each window as $\sigma$ and the range of each window as $R$. Then, assign the standard deviation and range value to all pixels in the window so that we can get four dispersion measurement images.

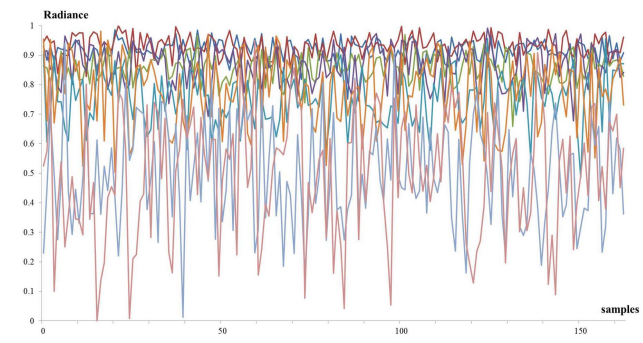

(a)

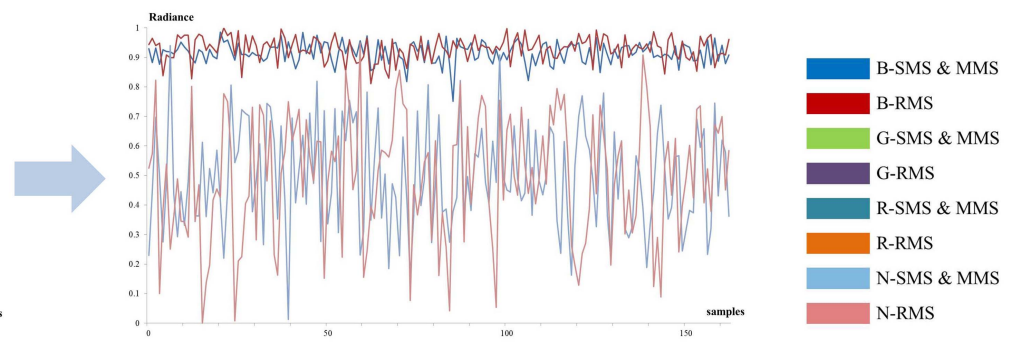

(b)

Figure 7. Band selection of high-resolution images. (a) The image consists of four bands (blue, green, red, and near infrared). The RMS has mixed spectral features with the other two types and quantities of redundant information that exist. (b) Little redundant information exists when there are only two bands left (blue and near infrared), which means less complexity and calculations of the algorithm.

It is worth mentioning that the $5 \times 5$ window in this step is the optimal traversal window selected by repeated experiments. The experimental results of the other windows are shown in Figure 8 and Table 2.

Table 2. Extraction accuracies of the RMS when using windows with different sizes.

\begin{tabular}{cccccc}
\hline Size & $\mathbf{3} \times \mathbf{3}$ & $\mathbf{4} \times \mathbf{4}$ & $\mathbf{5} \times \mathbf{5}$ & $\mathbf{6} \times \mathbf{6}$ & $\mathbf{7} \times \mathbf{7}$ \\
\hline OA $(\%)$ & 77.04 & 80.22 & 83.53 & 80.37 & 80.90 \\
\hline
\end{tabular}




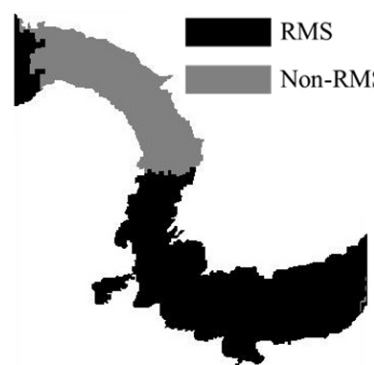

Groundtruth

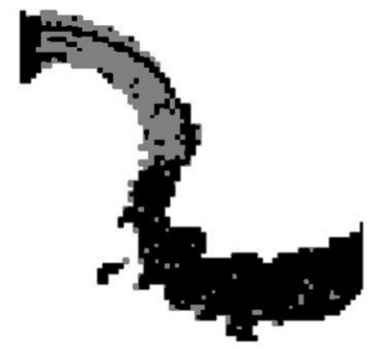

$5 \times 5$

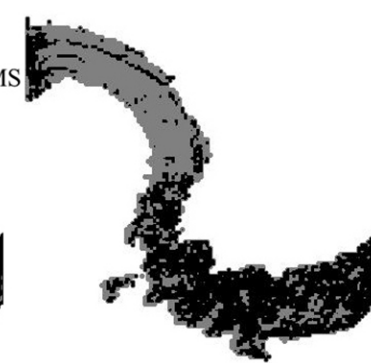

$3 \times 3$

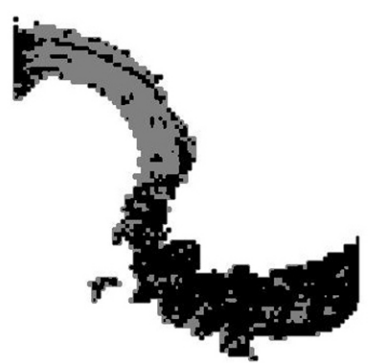

$4 \times 4$

\section{$\stackrel{N}{A}$}

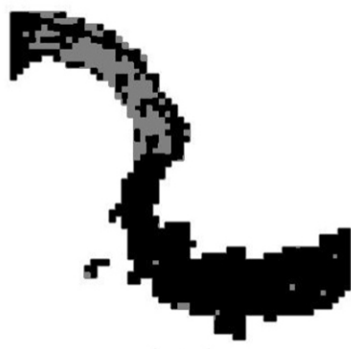

$6 \times 6$

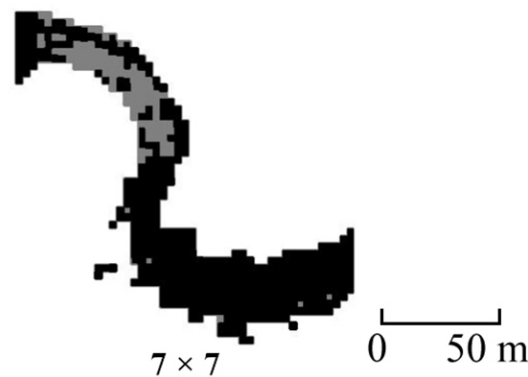

Figure 8. Extraction results of the RMS when using windows with different sizes.

(3) The four images were added together by influence weights to obtain the final measures of the dispersion image. In order to evaluate the contribution of each dispersion measurement image, the information entropy was estimated as

$$
E=\sum_{i=0}^{255} p_{i} \log p_{i}
$$

where $p_{i}$ is the probability of gray value $i$.

Then, the weights were calculated as

$$
W_{k}=\frac{E_{k}}{\sum_{k=1}^{4} E_{k}} .
$$

(4) The Marr edge detection operator was used in the final measures of the dispersion image to determine the RMS edge so that the RMS was extracted.

\subsection{Tidal Correction}

Influenced by tidal actions, tidal flat wetlands emerge differently in images at different moments of the same day. Thus, it was necessary to determine the low tide location for a more accurate extraction of tidal flat wetlands in actual demands. In order to predict the low tidal flat line of tidal flat wetlands, the slope of the tidal flat was calculated first according to the tidal height difference and the instant waterlines of one place at a different time. Then, the low tidal flat line of the wetlands was corrected to the low tide line.

The principle of tidal correction is shown in Figure 9, and the implication of each character is explained below the figure. By computing the tidal height difference between different time and the average distance of the instantaneous waterlines of two phases, the angle of the tidal flat slope $\theta$ is calculated as

$$
\theta=\arctan \frac{\Delta H}{\Delta L}
$$

According to the average low tide height $H_{\text {low }}$, the correction distance $L_{\text {low }}$, i.e., the low tide line is computed as

$$
L_{\text {low }}=\frac{h-H_{\text {low }}}{\tan \theta}
$$


where $h$ represents the tide height in the satellite transit time. After tidal correction, the results of the complete tidal flat wetlands could be obtained.

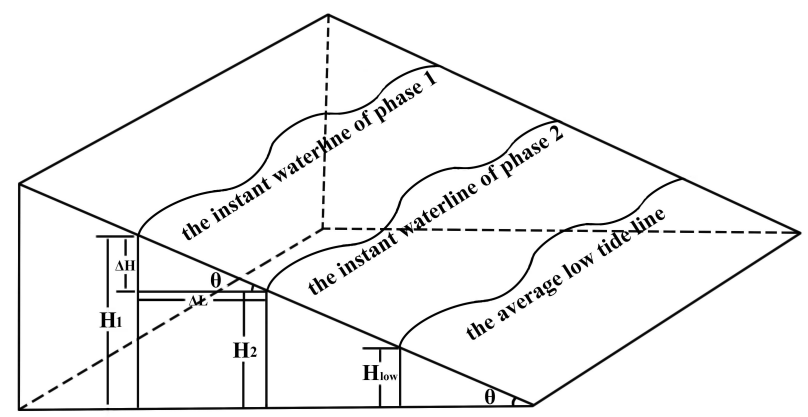

Figure 9. Schematic diagram of tidal correction. $\theta$ is the angle of the tidal flat slope. $H_{1}$ and $H_{2}$ represent the tide height at Time 1 and Time 2, respectively. $\Delta H$ is the tidal height difference between different times, and $\Delta L$ represents the average distance of the instantaneous waterlines of the two phases. $H_{\text {low }}$ is the average low tide height.

The tidal correction method is based on the assumption that the tidal flat slope is a gentle slope formed under the action of waves. The bottom matrix of the rocky coast is relatively hard, and the muddy beaches are mostly distributed around the lagoon. Therefore, these two types of tidal flat wetlands are less affected by the waves. The boundary of the low tide beach is to extract the water edge, so the tide level correction method in this study was mainly applied to the low tide beach boundary correction of sandy beaches. The key to the effectiveness of this method is whether the tidal flat slope is a gentle slope with a stable slope. In order to verify the correctness of this hypothesis, we first calculated the slope angle of the tidal flat based on the above principle, and then we obtained a high-resolution image of the third time phase of the same area and calculated the instantaneous waterline of that phase based on the slope angle and the imaging time of the third time phase image. We compared the actual extracted instantaneous water edge. If the two were similar, it indicated that the tidal flat slope angle remained unchanged; that is, the tide level correction method was effective.

To specifically evaluate the performance of tidal correction, the tidal flat slope was first calculated according to the information from Time 1 and Time 2. Then, the predicting instant waterline of the third time could be obtained on the basis of the tidal flat slope and the instant tide height, while the actual instant waterline could be acquired from the three previous extraction steps. Then, $\mathrm{m}$ sample points were chosen from the predicting instant waterline and the actual one. The root mean square error (RMSE), the mean absolute error (MAE), and the standard deviation (SD) between the two waterlines were calculated to evaluate their deviation quantitatively, expressed as

$$
\begin{gathered}
\text { RMSE }=\sqrt{\frac{1}{m} \sum_{i=1}^{m} d_{i}{ }^{2}} \\
M A E=\frac{1}{m} \sum_{i=1}^{m} d_{i} \\
S D=\sqrt{\frac{1}{m} \sum_{i=1}^{m}\left(d_{i}-d_{u}\right)^{2}}
\end{gathered}
$$

where $m$ represents the number of sample points, $d_{i}$ represents the Euclidean distance of point $i$ between two lines, and $d_{u}$ is the average value of $d_{i}$. 


\section{Results and Discussions}

\subsection{Research Data}

Six WorldView-2 images (Figure 10), located in Hengqin Island, Guangdong Province, China, respectively, acquired on 21 October 2015, 1 November 2017, and 15 January 2018, were utilized in this study. The Gram-Schmidt spectral sharpening method was used to fuse the multispectral bands and the panchromatic band, and then the cubic convolution method was used to resample the fusion images to an identical resolution. Detailed parameters of the used data are described in Table 3. Fusion images were finally used, containing red, green, blue, and near-infrared bands with a resolution of $0.5 \mathrm{~m}$. The sizes of Image 1 and Image 2 are 11,176 $\times 7466$ and $7446 \times 5631$ pixels, respectively.

Table 3. Detailed parameters of the used data.

\begin{tabular}{|c|c|}
\hline Name & Parameters \\
\hline Satellite & WorldView-2 (including multispectral and panchromatic sensors) \\
\hline The regression period & 1.1 days \\
\hline \multirow{4}{*}{ Original resolutions } & $\begin{array}{cc}\text { Wavelength (nm) } & 450-1040 \text { (panchromatic), } 450-510 \text { (blue), 510-580 (green), } \\
630-690 \text { (red), and 770-895 (NIR) }\end{array}$ \\
\hline & 2015.10.21 - 1.891 (multispectral) and 0.473 (panchromatic) \\
\hline & 2017.11.01 - 1.321 (multispectral) and 0.330 (panchromatic) \\
\hline & 2018.01.15 - 1.332 (multispectral) and 0.333 (panchromatic) \\
\hline Fusion method & Gram-Schmidt spectral sharpening method \\
\hline Resampling method & Cubic convolution method \\
\hline
\end{tabular}

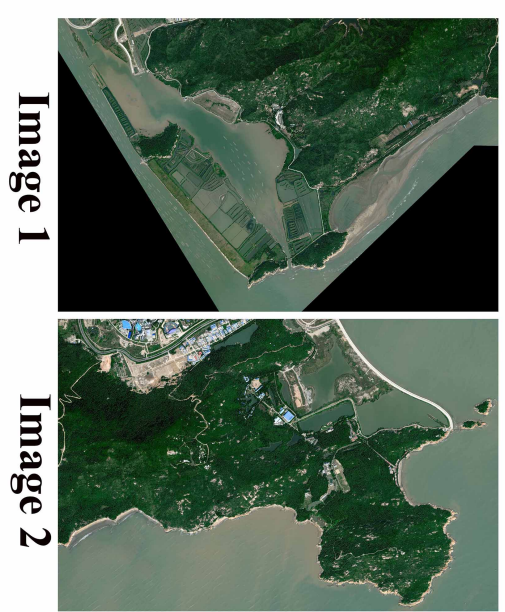

2015

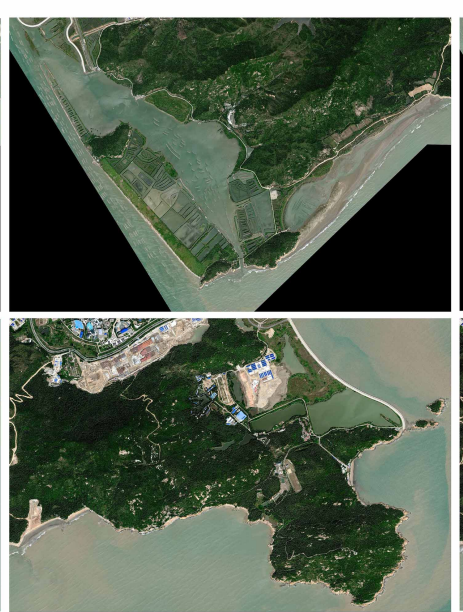

2017

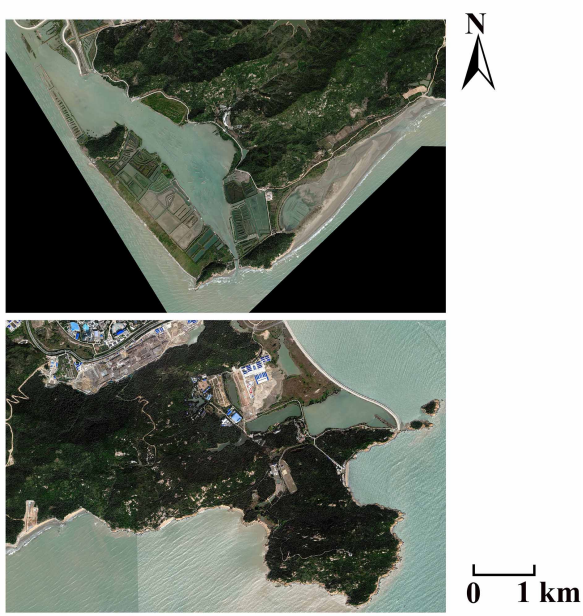

2018

Figure 10. Research images from three years.

\subsection{Performance of NSP}

According to the NSP algorithm, slope change rate curves of different shore polygon edges were calculated, and the results are shown in Figure 11. There is a shoreline with both natural features and artificial features in the test area in Figure 11a. It can be seen from the slope change rate curve that the slope fluctuates at the beginning and then becomes steady from a certain point. According to the different fluctuant characteristics of the slope change rates, it is clear that the left part of the curve comes from the natural excerpt, and the right part comes from the artificial one. Figure $11 \mathrm{~b}, \mathrm{c}$ demonstrates the slope change rate curve of an artificial shoreline and a natural shoreline, which express the same fluctuations as in Figure 11a. 


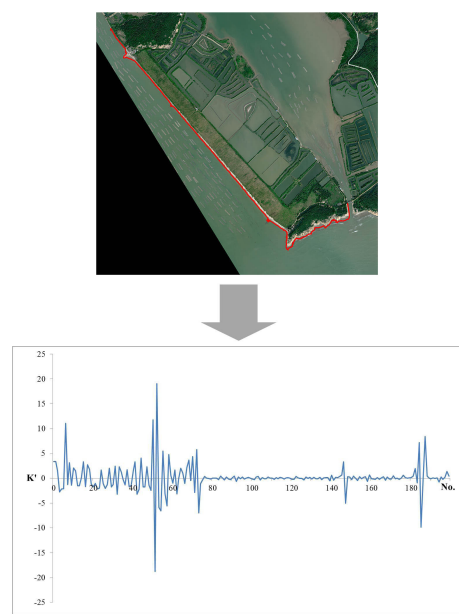

(a)

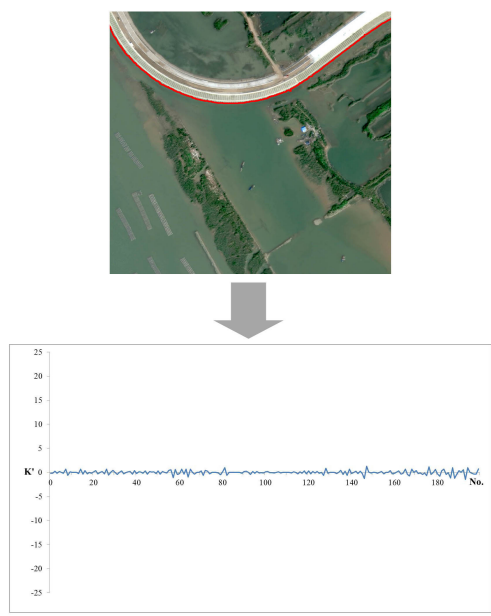

(b)

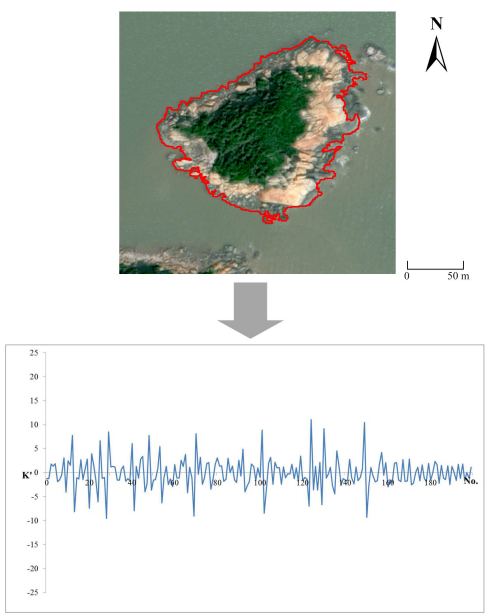

(c)

Figure 11. Examples of slope change rate curves from different shore polygon edges. (a) Slope change rate curves of the area with both the artificial shore and the natural shore. (b) Slope change rate curves of the artificial shore. (c) Slope change rate curves of the natural shore.

The NSP algorithm was executed on two images from 2015. The results are shown in Figure 12, and the evaluating data are listed in Table 4 . When the extraction results of Image 1 were compared with the ground truth, it can be seen from Figure 12a that most of the natural shores were extracted correctly, with only a small number of misextractions around the shore edges. Extraction accuracies and the kappa coefficient in Table 4 justify the analysis above. The extraction results of Image 2 in Figure $12 \mathrm{~b}$ are approximately correct, with a producer's accuracy of $88.00 \%$ and a user's accuracy of $93.53 \%$. However, it can be seen from Figure 12a that an unnatural shore on the far north side of Image 1 was mistakenly extracted as a natural shore due to its irregular edge. In Image 2, there are also some misextractions at the junction of the two shore types on the middle east side. The misextractions illustrate the limitations of the NSP algorithm in that it is excessively dependent on the shape of shore edges. In this condition, manual corrections will be needed according to practical applications. Despite some defects in these particular situations, the algorithm in this step performed well in the general prediction of natural shorelines.

Table 4. Extraction accuracies and the kappa coefficient after NSP.

\begin{tabular}{lcccc}
\hline & PA (\%) & UA (\%) & OA (\%) & $\kappa$ \\
\hline Image1 & 94.25 & 89.70 & 99.64 & 0.9161 \\
Image2 & 88.00 & 93.53 & 99.41 & 0.9050 \\
\hline
\end{tabular}

Image 1

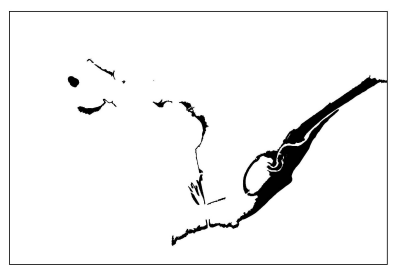

Groundtruth

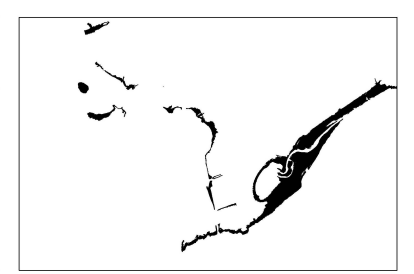

(a)
Image 2

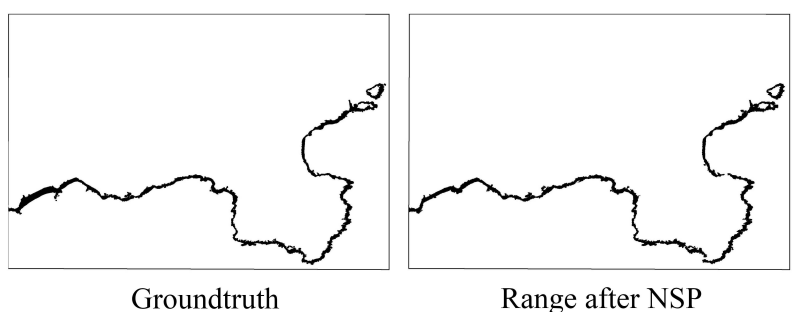

(b)

Figure 12. Results after NSP from two images in 2015. (a) Groundtruth and result of Image 1. (b) Groundtruth and result of Image 2. 


\subsection{Effectiveness of Tidal Correction}

In this study, images from 2015 and 2018 were, respectively, selected as the first and second time to calculate the tide flat slope, and images from 2017 were selected for evaluation. According to the method in Section 2.4, the instant waterline prediction results of the four typical experimental areas are illustrated in Figure 13, and the corresponding accuracy indexes are shown in Table 5.

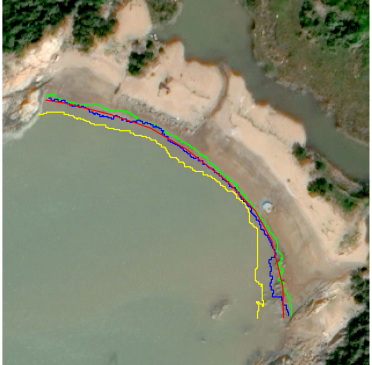

Area 1

Instantaneous shoreline of 2015

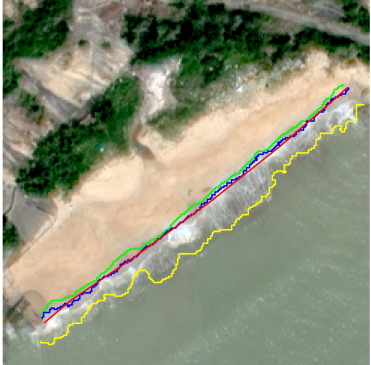

Area 2

Instantaneous shoreline of 2018

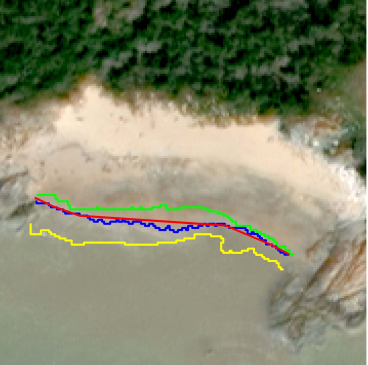

Area 3

Instantaneous shoreline of 2017

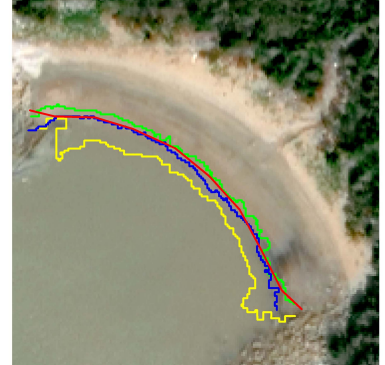

Area 4

Predicted shoreline of 2017

Figure 13. Tidal correction of four typical experimental areas.

In Figure 13, blue lines represent the actual extracted instant waterlines, and red lines represent the predicted ones according to the tidal correction method. In Area 1, most of the actual waterline and the predicted waterline coincide, but only small sections around the right endpoint deviate from each other. Observed from the image, we can see that there are rocks located in the sand marine shore, which could have prevented the sand shore from forming a gentle slope. Therefore, the terrain of the right shore does not conform to the hypothetical topography, resulting in a mistake. As shown in Area 2, Area 3, and Area 4 , the real waterlines coincide well with the predicted ones, and the accuracy indexes in Table 5 also confirm the consequent RMSE and MAE of less than 2 and the SD less than 0.6 , which verifies the performance of the tidal correction. When the tidal correction is conducted, each nonadjacent tidal flat wetland has to be processed independently, which means that low efficiency could be a defect of this method. Thus, we may consider parallel processing in future attempts.

Table 5. Tidal correction evaluation results.

\begin{tabular}{lccc}
\hline & RMSE & MAE & SD \\
\hline Area 1 & 1.98 & 1.70 & 2.41 \\
Area 2 & 1.08 & 0.97 & 0.38 \\
Area 3 & 1.28 & 1.14 & 0.58 \\
Area 4 & 1.88 & 1.81 & 0.58 \\
\hline
\end{tabular}

\subsection{Comparison with Other Methods}

In this section, the proposed method is applied to six experimental images from three years, and Figure 14 presents the results. It can be seen that the proposed method is able to delineate and classify most of the tidal flat wetlands and provide satisfactory results.

In order to fully verify the advantages of the proposed method, both classical machine learning methods and deep learning methods were considered as comparison methods. In terms of classical machine learning methods, no methods were specifically used for tidal flat wetland extraction; therefore, the support vector machine (SVM) method [24] and the random forest (RF) method [35], which performed better than the others in the situation of this research through experiments, were selected as comparison methods. In terms of deep learning methods, considering the suitable classification methods and the hash 
rate of our computers comprehensively, the ResNet34 model [36] was selected as the third comparison method.
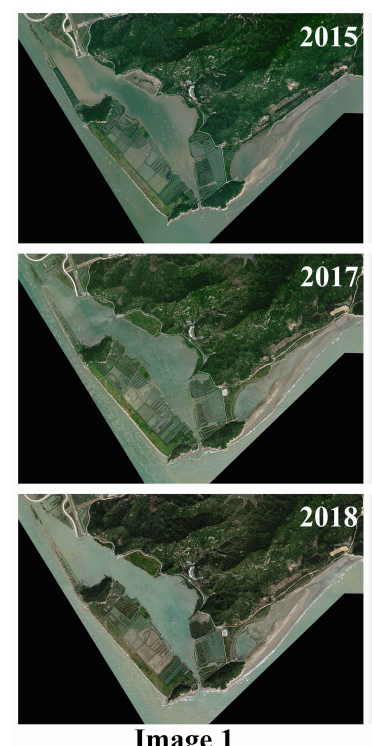

Image 1
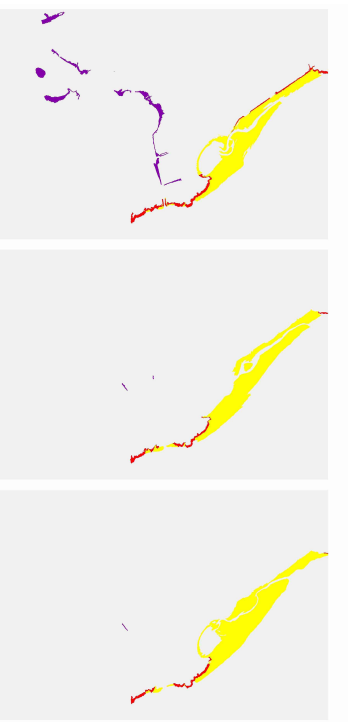

Extraction results
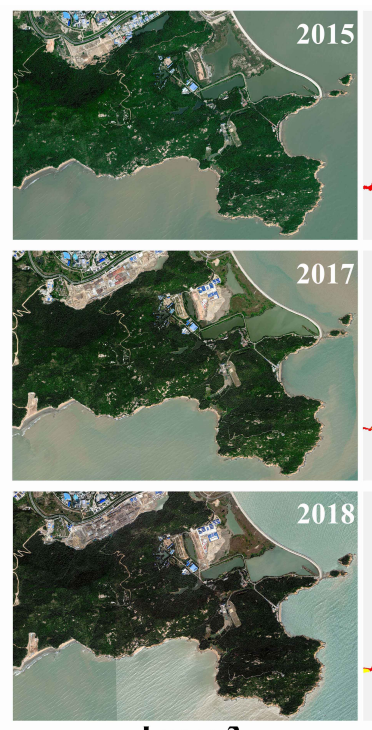

Image 2
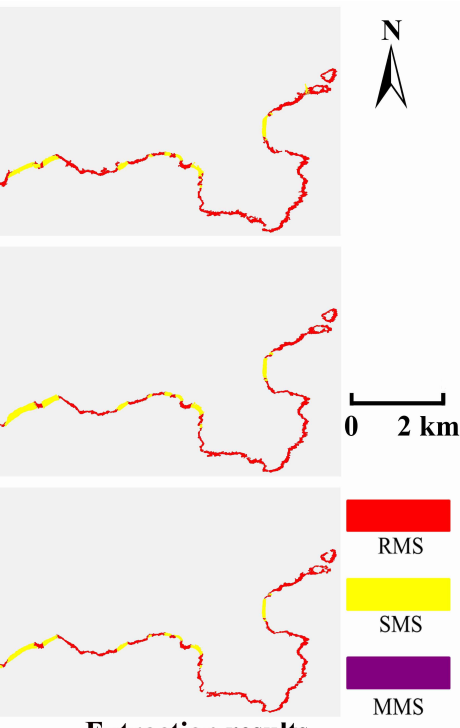

Extraction results

Figure 14. Tidal flat wetlands extraction results of the proposed method.

In order to guarantee the same training samples, FNEA segmentation was conducted on the research images from October 21, 2015, at first, sharing the same parameters of FNEA with the proposed method (detailed values are clarified in Section 2.1.1). Samples of each tidal flat wetland category (in the form of objects) were obtained by manual interpretation and treated as the training samples of ResNet34. Then, the object-based samples were transformed into pixel-based ones, which were considered as the training samples of the SVM and RF. Finally, 63 objects in Image 1 and 77 objects in Image 2 were selected as training samples. Moreover, all the comparison methods were conducted from the perspective of logical layering (the same initial classification and geographical analysis), which guaranteed a fair comparison. To quantify the performance values, the overall accuracy (OA) and kappa (k) were selected to evaluate the four methods. There are 400 and 350 verification points in Image 1 and Image 2, respectively, for the experimental data from each year.

Figure 15a-f provides a visual comparison of the different methods, and the corresponding statistical results are listed in Table 6. For the SVM method, land and sea are separated in Image 1 from all three years, which mainly depends on the results of the initial classification in this study. There are still misclassifications when observing the details: the RMS and the SMS on the south coast are not distinguished, the RMS and the MMS around the lagoon are mixed, and the white waves in the sea are also misclassified into tidal flat wetlands due to their similar spectral features. The results of the SVM in Image 2 are a little more reliable than those in Image 1 when noticing the OA, which are caused by fewer tidal flat wetland categories, but it still shows a large number of misclassifications from the visual results. Moreover, the SVM method cannot distinguish between the artificial boundaries and the natural boundaries; for example, the artificial roads in the northeast corner of Image 2 are misclassified as natural targets. The extraction results of the RF method contain too much "salt-and-pepper noise" just as the SVM, despite the fact that it performs better than the SVM in accuracies, which are caused by their pixel-based classification modus. The ResNet34 model is conducted in an object-based way, just as the proposed one, which can solve the noise problems. Observing the visual results, we can clearly notice that the last two object-based methods perform well in achieving the purity of results. For ResNet34, there are also misclassifications between artificial boundaries and natural boundaries: the artificial roads in the northwest corner and the artificial coast on the west 
are mistaken for tidal flat wetlands in Image 1 from all three years. Additionally, ResNet34 performs poorly when distinguishing the RMS from the other two kinds. Large sample quantity reliance is the main limitation of deep learning methods, although there are only a small number of samples in this study that caused unsatisfying results when ResNet34 was unable to learn enough features of tidal flat wetlands. It can be concluded that the ResNet34 model has limitations when extracting tidal flat wetlands from high-resolution images when there are not enough samples. With the same samples, the proposed method performs well when solving misclassification problems, which can be seen from the results. The proposed NSP algorithm distinguishes the misclassifications of artificial objects from natural objects, and the RSD feature descriptor can easily extract the RMS when it has similar spectral characteristics with the surroundings. It can be seen from Table 6 that the OA of the proposed method are all above $88 \%$, exceeding the three compared methods.

Concluding the comparison results, the pixel-based SVM and RF methods cause "saltand-pepper noise" and misclassifications when extracting tidal flat wetlands. The deep learning model solves the noise problem, while it still shows unsatisfying results with insufficient samples. The proposed method performs well in these aspects despite its lower processing speed. Additionally, there is no other efficient method with similar accuracy to the proposed one, which means an excellent extraction consequence is more significant, despite the fact that there are complex steps in the proposed method. Therefore, the proposed method has particular advantages in the extraction of tidal flat wetlands from high-resolution images.

(a)
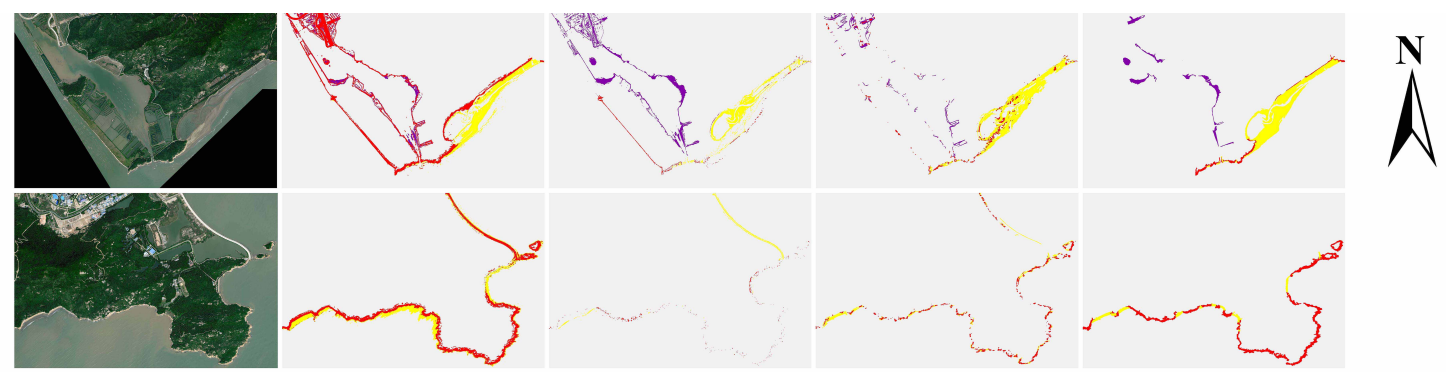

(c)
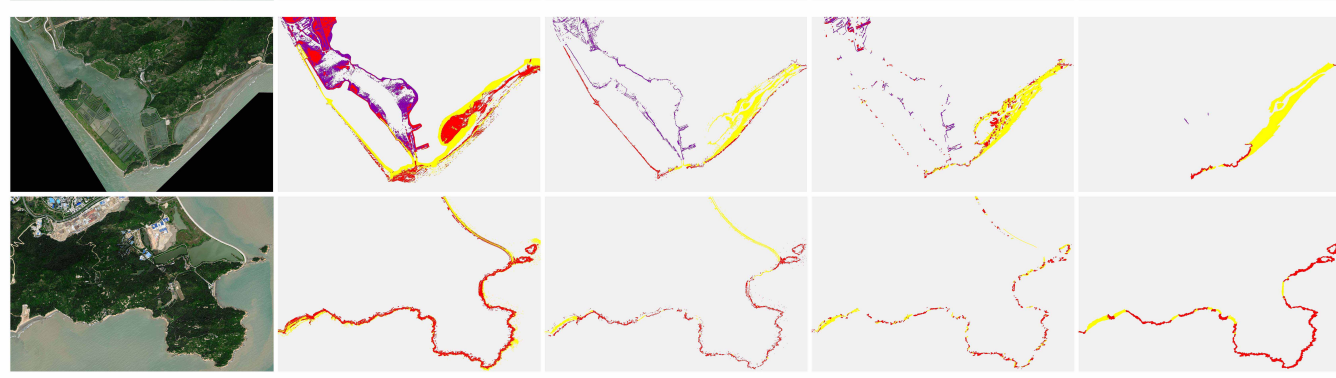

(d)

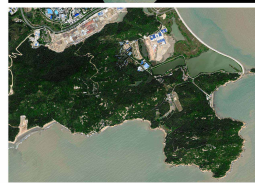

(e)

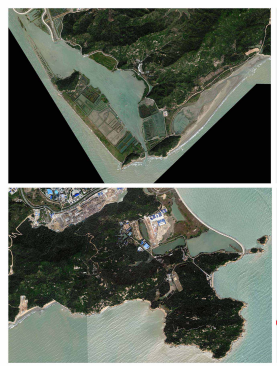

Images

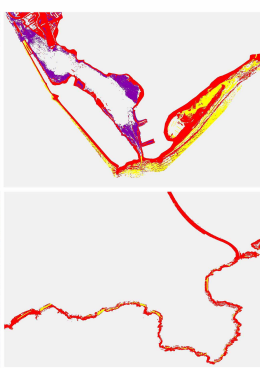

SVM

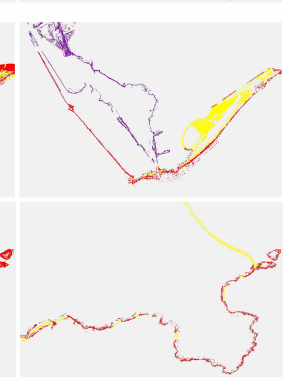

RF

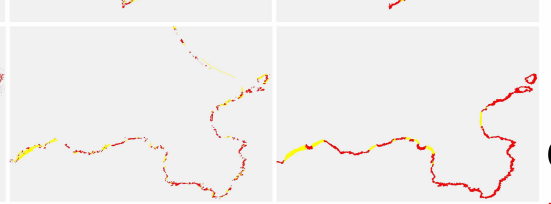

$5 \quad 5$ km

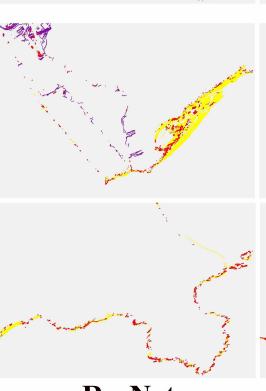

ResNet

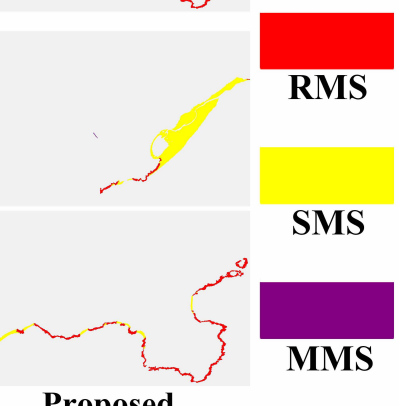

Figure 15. Comparison of results with other methods: (a) results of Image 1 in 2015; (b) results of Image 2 in 2015; (c) results of Image 1 in 2017; (d) results of Image 2 in 2017; (e) results of Image 1 in 2018; (f) results of Image 2 in 2018. 
Table 6. Comparison of the accuracy of the four wetland classification methods.

\begin{tabular}{lccccccccc}
\hline & \multicolumn{2}{c}{ SVM } & \multicolumn{2}{c}{ RF } & \multicolumn{2}{c}{ ResNet } & \multicolumn{2}{c}{ Proposed } \\
\hline & Time & OA (\%) & $\kappa$ & OA (\%) & $\kappa$ & OA (\%) & $\kappa$ & OA (\%) & $\kappa$ \\
\hline \multirow{2}{*}{ Image 1 } & 2015 & 51.25 & 0.34 & 66.50 & 0.51 & 53.25 & 0.37 & 88.50 & 0.84 \\
& 2017 & 53.00 & 0.10 & 69.25 & 0.46 & 75.00 & 0.46 & 92.75 & 0.84 \\
& 2018 & 53.75 & 0.28 & 64.75 & 0.33 & 76.00 & 0.55 & 93.50 & 0.87 \\
\hline \multirow{3}{*}{ Image 2 } & 2015 & 71.43 & 0.49 & 44.86 & 0.24 & 57.71 & 0.37 & 96.29 & 0.93 \\
& 2017 & 72.29 & 0.52 & 63.14 & 0.44 & 57.43 & 0.35 & 95.71 & 0.92 \\
& 2018 & 76.57 & 0.58 & 73.14 & 0.56 & 48.00 & 0.24 & 92.86 & 0.87 \\
\hline
\end{tabular}

\section{Conclusions}

In this study, a comprehensive method for tidal flat wetland extraction from highresolution images was proposed. First, the decision tree method based on remote sensing features and geographical buffers was constructed for the initial classification. Next, an NSP algorithm based on the slope change rate was designed to refine the range of the tidal flat wetland. Then, a range and standard deviation descriptor was constructed to extract the RMS, and a geographical analysis method was considered to distinguish the SMS and MMS. Finally, a tidal correction strategy was introduced to regulate the borderline of tidal flat wetlands. The results showed that the proposed method can achieve good performance for the extraction of tidal flat wetlands from high-resolution images. By the delineation and classification method, the distribution of tidal flat wetlands can be investigated easily, consequently contributing to wetland protection.

A defect still comes from the strong correlation between the multiple steps, which means that the results of the previous step will affect the results of the following step directly. Therefore, emphasis will be placed on optimizing the accuracy in each step in the future. In addition, we will try to expand this method to more complex shore situations in order to increase the versatility.

Author Contributions: Conceptualization, Hong Pan and Yonghong Jia; Data curation, Hong Pan, Dawei Zhao, Tianyu Xiu and Fuzhi Duan; Formal analysis, Hong Pan; Investigation, Hong Pan, Dawei Zhao, Tianyu Xiu and Fuzhi Duan; Methodology, Hong Pan; Supervision, Yonghong Jia; Validation, Hong Pan; Writing — original draft, Hong Pan; Writing — review and editing, Hong Pan and Yonghong Jia. All authors have read and agreed to the published version of the manuscript.

Funding: This research was funded by Development Fund of the National Surveying and Mapping Science and Technology.

Institutional Review Board Statement: Not applicable.

Informed Consent Statement: Not applicable.

Data Availability Statement: Data or models support the findings of this study are available from the corresponding author upon reasonable request.

Conflicts of Interest: The authors declare no conflict of interest.

\section{References}

1. Batzer, D.P. Wetland ecology: Principles and conservation. Wilson Bull. 2001, 113, 354-361. [CrossRef]

2. Cahoon, D.R.; Hensel, P.F.; Spencer, T.; Reed, D.J.; Saintilan, N. Coastal Wetland Vulnerability to Relative Sea-Level Rise: Wetland Elevation Trends and Process Controls. In Wetlands and Natural Resource Management; Springer: Berlin/Heidelberg, Germany, 2006.

3. Bellio, M.; Kingsford, R.T. Alteration of wetland hydrology in coastal lagoons: Implications for shorebird conservation and wetland restoration at a Ramsar site in Sri Lanka. Biol. Conserv. 2013, 167, 57-68. [CrossRef]

4. David, M.M.; Meli, P.; Maria, I.V.R.; Aronson, J. Ecosystem response to interventions: Lessons from restored and created wetland ecosystems. J. Appl. Ecol. 2015, 52, 1528-1537.

5. Klemas, V. Remote Sensing of Riparian and Wetland Buffers: An Overview. J. Coast. Res. 2014, 297, 869-880. [CrossRef]

6. Kuklinski, P. Ecology of stone-encrusting organisms in the Greenland Sea-A review. Polar Res. 2009, 28, 222-237. [CrossRef]

7. Ghosh, A.; Joshi, P.K. Assessment of pan-sharpened very high-resolution WorldView-2 images. Int. J. Remote Sens. 2013, 34, 8336-8359. [CrossRef] 
8. Pu, R.; Landry, S. A comparative analysis of high spatial resolution IKONOS and WorldView-2 imagery for mapping urban tree species. Remote Sens. Environ. 2012, 124, 516-533. [CrossRef]

9. Carle, M.V.; Wang, L.; Sasser, C.E. Mapping freshwater marsh species distributions using WorldView-2 high-resolution multispectral satellite imagery. Int. J. Remote Sens. 2014, 35, 4698-4716. [CrossRef]

10. Li, H.; Wang, Y.; Xiang, S.; Duan, J.; Zhu, F.; Pan, C.; Com, H. A label propagation method using spatial-spectral consistency for hyperspectral image classification. Int. J. Remote Sens. 2016, 37, 191-211. [CrossRef]

11. Lahet, F.; Ouillon, S.; Forget, P. Colour classification of coastal waters of the Ebro river plume from spectral reflectances. Int. J. Remote Sens. 2001, 22, 1639-1664. [CrossRef]

12. Kong, D.; Xu, J.; Yin, J.; Yan, H. Classification of MODIS images combining surface temperature and texture features using the Support Vector Machine method for estimation of the extent of sea ice in the frozen Bohai Bay, China. Int. J. Remote Sens. 2015, 36, 2734-2750.

13. Defries, R.S.; Townshend, J.R.G. NDVI-derived land cover classifications at a global scale. Int. J. Remote Sens. 1994, 15, 3567-3586. [CrossRef]

14. Wu, J.; Liu, Y.; Wang, J.; Ting, H. Application of Hyperion data to land degradation mapping in the Hengshan region of China. Int. J. Remote Sens. 2010, 31, 5145-5161. [CrossRef]

15. Benferhat, S.; Boudjelida, A.; Tabia, K.; Drias, H. An intrusion detection and alert correlation approach based on revising probabilistic classifiers using expert knowledge. Appl. Intell. 2013, 38, 520-540. [CrossRef]

16. Muchoney, D.; Borak, J.; Chi, H.; Friedl, M.; Gopal, S.; Hodges, J.; Morrow, N.; Strahler, A. Application of the MODIS global supervised classification model to vegetation and land cover mapping of Central America. Int. J. Remote Sens. 2000, 21, 1115-1138. [CrossRef]

17. Zhou, Y.; Xiao, X.; Qin, Y.; Dong, J.; Zhang, G.; Kou, W.; Jin, C.; Wang, J.; Li, X. Mapping paddy rice planting area in rice-wetland coexistent areas through analysis of Landsat 8 OLI and MODIS images. Int. J. Appl. Earth Obs. Geoinf. 2016, 46, 1-12. [CrossRef]

18. Xiao, X.; Boles, S.; Liu, J.; Zhuang, D.; Frolking, S.; Li, C.; Salas, W.; Moore, I.B. Mapping paddy rice agriculture in southern China using multi-temporal MODIS images. Remote Sens. Environ. 2005, 95, 480-492. [CrossRef]

19. Lyons, M.B.; Keith, D.A.; Phinn, S.R.; Mason, T.J.; Elith, J. A comparison of resampling methods for remote sensing classification and accuracy assessment. Remote Sens. Environ. 2018, 208, 145-153. [CrossRef]

20. Raju, P.V. Classification of wheat crop with multi-temporal images: Performance of maximum likelihood and artificial neural networks. Int. J. Remote Sens. 2003, 24, 4871-4890.

21. Carr, J.R. Spatial Statistics for Remote Sensing. Math. Geosci. 2005, 37, 549-550. [CrossRef]

22. Hearst, M.A.; Dumais, S.T.; Osman, E.; Platt, J.; Scholkopf, B. Support vector machines. IEEE Intell. Syst. 1998, 13, 18-28. [CrossRef]

23. Yu, L.; Porwal, A.; Holden, E.J.; Dentith, M.C. Towards automatic lithological classification from remote sensing data using support vector machines. Comput. Geosci. 2012, 45, 229-239. [CrossRef]

24. Maulik, U.; Chakraborty, D. A self-trained ensemble with semisupervised SVM: An application to pixel classification of remote sensing imagery. Pattern Recognit. 2011, 44, 615-623. [CrossRef]

25. Friedl, M.A.; Brodley, C.E. Decision tree classification of land cover from remotely sensed data. Remote Sens. Environ. 1997, 61, 399-409. [CrossRef]

26. Pal, M.; Mather, P.M. An assessment of the effectiveness of decision tree methods for land cover classification. Remote Sens. Environ. 2003, 86, 554-565. [CrossRef]

27. Hinton, G.E.; Osindero, S.; Teh, Y.W. A fast learning algorithm for deep belief nets. Neural Comput. 2017, 18, 1527-1554. [CrossRef]

28. Lin, L.; Dong, H.; Song, X. DBN-Based Classification of Spatial-Spectral Hyperspectral Data; Springer: Cham, Switzerland, 2017.

29. Qayyum, A.; Malik, A.S.; Saad, N.M.; Iqbal, M.; Abdullah, M.F.; Rasheed, W. Scene classification for aerial images based on CNN using sparse coding technique. Int. J. Remote Sens. 2017, 38, 2662-2685. [CrossRef]

30. Shunping, J.; Chi, Z.; Anjian, X.; Yun, S.; Duan, Y. 3D Convolutional Neural Networks for Crop Classification with Multi-Temporal Remote Sensing Images. Remote Sens. 2018, 10, 75.

31. Han, X.; Zhong, Y.; Zhao, B.; Zhang, L. Scene classification based on a hierarchical convolutional sparse auto-encoder for high spatial resolution imagery. Int. J. Remote Sens. 2017, 38, 514-536. [CrossRef]

32. Othman, E.; Bazi, Y.; Alajlan, N.; Alhichri, H.; Melgani, F. Using convolutional features and a sparse autoencoder for land-use scene classification. Int. J. Remote Sens. 2016, 37, 1977-1995. [CrossRef]

33. Gao, B. NDWI-a normalized difference water index for remote sensing of vegetation liquid water from space. Remote Sens. Environ. 1996, 58, 257-266. [CrossRef]

34. Huete, A.; Didan, K.; Miura, T.; Rodriguez, E.P.; Gao, X.; Ferreira, L.G. Overview of the radiometric and biophysical performance of the MODIS vegetation indices. Remote Sens. Environ. 2002, 83, 195-213. [CrossRef]

35. Breiman, L. Random forest. Mach. Learn. 2001, 45, 5-32. [CrossRef]

36. He, K.; Zhang, X.; Ren, S.; Jian, S. Deep Residual Learning for Image Recognition. In Proceedings of the 2016 IEEE Conference on Computer Vision and Pattern Recognition (CVPR), Las Vegas, NV, USA, 27-30 June 2016; pp. 770-778. 